\title{
An Integrated Modeling Method Supporting Product Development Process Optimization
}

\author{
Min $\mathrm{Li}^{1}$, Xiansheng Qin ${ }^{2}$ and Yabin $\mathrm{Xu}^{2}$ \\ 'School of Mechatronics, KunMing University of Science \& Technology, Kunming 650093, \\ P.R. China leah163@163.com \\ ${ }^{2}$ School of Mechatronics, Northwestern Polytechnical University, Xi' an 710072, P.R. China \\ xsqin@nwpu.edu.cn yabinxu@nwpu.edu.cn
}

\begin{abstract}
Constructing an effective process module is groundwork for product development process optimization. This paper imposes an activity/process based modeling idea which supports the integrated management and optimization of process development process. This idea uses the activity/process as the modeling basic cell. At first, the activity/process based PDP modeling theory is studied; Second, the product Development process Modeling based on the Process/Action (DMP/A) method is forwarded. The basic elements and their properties are defined, then the steps of DMP/A are given in the paper. Through integrating the definition and description of process structure and activity/process attribute, input and output, the process model by DMP/A method helps to programmer, supervise and control the product development activities/processes, as well as communicate messages with each other. This provides a supporting tool for process development process integrated optimization.
\end{abstract}

Keywords: Product life cycle systems, Life cycle process, Business process modeling, Modeling and description languages, Activity/Process

\section{INTRODUCTION}

Product development process (PDP) should be geared to the needs of manufacturing globalization and normalization. Manufacturing enterprises had employed some advanced manufacturing system theory and methods, such as Concurrent Engineering (CE) and Agile Manufacturing (AM), for the improvement and optimization of the product development process (PDP). These theory and methods are based on the analysis, operation and control of the business and product development process. It founded on PDP modeling. A good modeling method should support the application of advanced manufacturing theories and technologies like CE. Therefore, the study of process modeling is fundamental and significant for the mode optimization of advanced manufacturing system, the application and realization of advanced manufacturing technologies and the performance indexes improvement of product development system [1]. 
Process modeling should support process integration. This means not only the integration and share of process information, but also the cooperation, optimization and management of process. So, new modeling technology is needed to support effectively the process programming and management [2]. The existed research works mostly have no overall consideration of product, process information and process management. For solving this problem, this paper studies the integration of process modeling technology and contemporary PDP management methods to promote the application of PDP model, based on the comprehensive consideration of product, process information and process management.

\section{PROBLEMS PDP MODELING FACES}

Product development is a complicated process which involves a great deal of activities, information, personnel and resources. The collection of information and management of processes are two key factors among them. In PDP, activities are interrelated. Some activities execute with provision and support of many other related activities. Information of activities is dispersive and dynamic, and executants of activity often do not know which activities information their activity produced will influence. Then how to send the activity information to the product developer and process manager accurately and promptly is a problem PDP faces. In PDP, a tool that manages the activity information is cried for, and it is the goal of process modeling in this paper.

Another problem in PDP is how to support the cooperation among activities and process management under the environment of networks [3]. Because product development system is a very complex and gigantic system with non-linearity, local disorder may trigger chaos in the overall PDP, even the decline of system's whole performance. To manage and control such a system scientifically, an applicable model should be established at first. In other words, an operable and controllable system model or process model is needed to manipulate and analyze the system or process dynamically.

Process model supports mainly the process execution and process management. Process model could describe PDP more accurate than pure language and concept. With a describing and supporting tool of process, developer may simulate PDP, and then acquire information needed of PDP via communication among processes; manager may design and program PDP and supervise the progression and cost situation of processes. Furthermore, based on the process model, simulator and analyzer could be used to improve and optimize PDP.

\section{BASIC THEORY OF PDP INTEGRATED MODELING}

\subsection{Activity/Process Definition}


Process and activity are two basic concepts in process modeling. Usually process is regarded as the set of activities and relation among them, and activity is abstraction of product development task; process emphasizes relation and activity emphasizes independence. Their definition is as follow:

\subsubsection{Process Definition}

$$
P=(A, R)
$$

$P$ represents a process, and A represents the set of activities within process $P$. $R$ represents the set of relation among activities within process $P$, which called process structure. In the following equation, $n$ is the number of activities within process $P$.

$$
A=\{A[i], i=1,2 \ldots n, n \in N, n>1\}
$$

\subsubsection{Activity Definition}

An activity could be expressed by its input, output, items and its attribute state space (Figure 1). Activity A could be expressed as:

$$
A[i]=\{I[i], O[i], A C[i], A R[i]\}
$$

I [i] represents the set of $A[i]$ 's inputs; $O[i]$ represents the set of $A[i]$ 's outputs; $A C$ [i] represents the set of A[i]'s items; AR [i] represents the set of A[i]'s attributes state space which includes activity attributes and activity item attribute. At a given time, activity attributes and activity item attribute have given value, then these values compose matrix ar [i]. ar [i] is an element in state space AR [i] which called an attribute state.

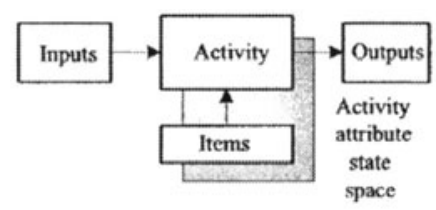

Figure 1. Expression of an Activity

\section{Set of inputs: $I$ [i]}

Set of inputs, 1 [i], could be divided into two kinds: IC[i] and ID[i]. IC[i] is the inputs with control attribute and $\mathrm{ID}[\mathrm{i}]$ is the inputs without control attribute. The difference between them is that inputs in IC [i] may cause the change of activity's state.

2. Set of outputs: $\mathrm{O}[\mathrm{i}]$

Like I [i], set of outputs, $O[i]$, could be divided into two kinds: $O C[i]$ and $O D[i]$. $O C[i]$ is the outputs with control attribute and $O D[i]$ is the outputs without control attribute. The difference between them is that outputs in $\mathrm{OC}[\mathrm{i}]$ are caused by change of activity's state.

In a system, elements interact by three ways. They are exchange of material, information and energy. PDP has the property of system. Activities in PDP interact by these three ways. The interaction is activity's input and output. Because material 
exchange and energy exchange could be described by information, set of inputs I[i] and set of outputs $\mathbf{O}$ [i] of activity $A$ [i] could be described by information.

3. Set of items: AC [i]

Set of items, $\mathrm{AC}$ [i], represents the $\mathrm{A}[\mathrm{i}]$ related object entity which is non-activity, non-input and non-output. In PDP, activity items include mainly organization and resources.

$$
\mathrm{AC}[\mathrm{i}]=\{\mathrm{AG}[\mathrm{i}], \mathrm{U}[\mathrm{i}]\}
$$

AG[i]: Organization, which is the organizational structure of activity;

$\mathrm{U}[\mathrm{i}]$ : Resources, which is the equipments and tools needed by activity execution.

04 . Attribute state space: AR [i]

$A R[i]$ represents the attribute state space of activity $A[i]$ (Table 1 ).

$$
\text { AR [i] }=[\text { RAR[i], RAG[i], RU [i], RI [i], RO [i]] }
$$

Table 1. Attribute State Space of Activity A [i]

\begin{tabular}{|l|l|l|l|l|l|l|l|l|l|}
\hline attribute state space & name & type & $\begin{array}{l}\text { Func- } \\
\text { tion }\end{array}$ & site & $\begin{array}{l}\text { start- } \\
\text { time }\end{array}$ & $\begin{array}{l}\text { end- } \\
\text { time }\end{array}$ & state & $\begin{array}{l}\text { Requi- } \\
\text { rement }\end{array}$ & cost \\
\hline activity RAR[i] & $\sqrt{ }$ & $\sqrt{ }$ & $\sqrt{ }$ & $\sqrt{ }$ & $\sqrt{ }$ & $\sqrt{ }$ & $\sqrt{ }$ & & $\sqrt{ }$ \\
\hline organizationRAG[i] & $\sqrt{ }$ & $\sqrt{ }$ & $\sqrt{ }$ & & & & $\sqrt{ }$ & & \\
\hline resource RU [i] & $\sqrt{ }$ & $\sqrt{ }$ & $\sqrt{ }$ & & & & $\sqrt{ }$ & $\sqrt{ }$ & \\
\hline input RI [i] & $\sqrt{ }$ & $\sqrt{ }$ & & & $\sqrt{ }$ & $\sqrt{ }$ & $\sqrt{ }$ & $\sqrt{ }$ & \\
\hline output RO [i] & $\sqrt{ }$ & $\sqrt{1}$ & & & $\sqrt{ }$ & $\sqrt{ }$ & $\sqrt{ }$ & $\sqrt{ }$ & \\
\hline
\end{tabular}

\subsubsection{Activity Output Function}

Activity output function $\mathrm{g}$ is a mapping which represents the transform of $\mathrm{I}[\mathrm{i}]$ to $O[i]$ when the activity is under the attribute state ar [i]. Then activity output

$$
\mathrm{O}[\mathrm{i}]=\mathrm{g}(\mathrm{I}[\mathrm{i}], \operatorname{ar}[\mathrm{i}]), \operatorname{ar}[\mathrm{i}] \in \mathrm{AR}[\mathrm{i}]
$$

\subsection{Relation among Activities}

To analyze the relation among activities with an eye to time, the following equation expresses relation between activity $A_{i}$ and $A_{j}$ :

$$
f\left(\operatorname{ar}[i](t), R^{\prime}{ }_{i j}(t), \operatorname{ar}[j](t)\right)=0, \operatorname{ar}[i], \operatorname{ar}[j] \in A R[j]
$$

$\operatorname{ar}[i](t)$ and $\operatorname{ar}[j](t)$ represent separately an attribute state of AR [i] and AR [j] at the moment $t$. $R^{\prime}$ ij represents the activity relation caused by $\operatorname{ar}[i](t)$ and $\operatorname{ar}[j](t)$.

We know from equation (7) that relation equation is decided by the impersonal rules of process. It is objective thing and determinate to any process. But because of the limitation of the knowledge of product, enterprise and society, we could not work out the relation equation of every process. The management and control of PDP requires participation of human being because of the complexity of process environment. 
In equation (7), when any two variables of $\operatorname{ar}[i](t), R^{\prime}{ }_{i j}(t)$ and $\operatorname{ar}[j](t)$ is given, the other one variable is decided by the corresponding relation equation.

\subsection{Process Structure Definition}

Process structure $\mathrm{R}$ is the abstract of relation among activities. Under given environment, if there exists relation $R_{i j}(t)(1 \leq i \leq n, \quad 1 \leq j \leq n ; i \neq j, n>1)$ in the moment $t$ between activity $A_{i}$ and $A_{j}$ in process $P$, then the set of all relation $R_{j j}(t)(1 \leq i \leq n$, $1 \leq j \leq n ; \quad i \neq j, n>1)$ is called process structure, $R(t)$.

$$
R(t)=\left\{R_{i j}(t) \mid 1 \leq i \leq n, 1 \leq j \leq n ; i \neq j, n>1\right\}
$$

From definitions in equation (7) and (8), we know that various activity attributes show various relation. The progression attribute of activity shows certain activity relation, and resource attribute shows another activity relation. This leads to the difference among process expression. A uniform process modeling method is needed.

\subsection{Breaking Activity/Process Down}

PDP expansion simplifies the process research, so method breaking process down layer by layer is employed to describe activity/process (Figure 2). Activity/process is broken down from up to bottom and more detailed step by step. Each sonactivity/process is regarded as a local and independent module, and then it has inputs, outputs, control information, task and goal etc., and has certain duties, attributes and behaviors as its father-activity/process.

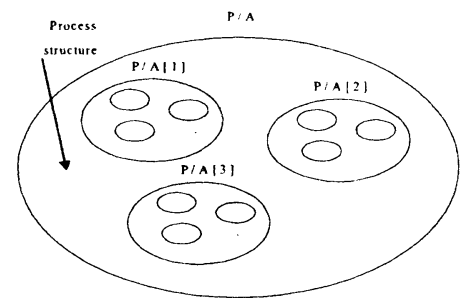

Figure 2. Breaking Down Activity/Process

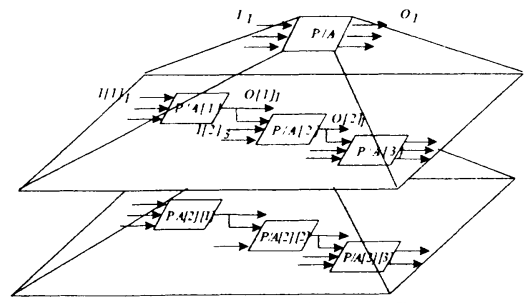

Figure 3. Breaking Down Inputs and Outputs of Activity/Process

\subsection{Breaking down Inputs and Outputs of Activity/Process}

The key point of activity/process management is to manage the relation among activities. Activity attribute state space is the foundation of activity relation. Inputs and outputs of activity show the task relation and information, material flow relation, on which other activity relation based. In process model, if inputs and outputs of an activity is defined, then they could be broken down and their attributes, including time, quality standard and type, etc., could be endued with. Moreover, all kinds of 
activity relation could be programmed and managed in the light of activity attributes and relation between inputs and outputs.

In process model, inputs and outputs of activity/process could be described by information. As the deployment of activity/process, a large, fuzzy input (output) is gradually transferred into those detailed input (output) information (Figure 3).

If breaking activity/process, $\mathrm{P} / \mathrm{A}[\mathrm{i}]$, down as follow:

$$
P / A[i]=(\{P / A[i][j], j=1,2 \ldots n, n \in N, n>1\}, R[i])
$$

$n$ is the number of sub-activity/process, $P / A[i][j]$, in $P / A[i] ; R[i]$ represents the process structure of $\mathrm{P} / \mathrm{A}[\mathrm{i}]$.

If there is no coupling between input, $\mathrm{I}[\mathrm{i}]$, and output, $\mathrm{O}[\mathrm{I}]$, of activity/process $\mathrm{P} / \mathrm{A}[\mathrm{i}]$, then

$$
\begin{aligned}
& I[i]=\sum_{j=1}^{n} I[i][j]-\sum_{j=1}^{n}\left(I[i][j] \cap \sum_{k=1, k \neq j}^{n} O[i][k]\right) \\
& O[i]=\sum_{j=1}^{n} O[i][j]-\sum_{j=1}^{n}\left(O[i][j] \cap \sum_{k=1, k \neq j}^{n} I[i][k]\right)
\end{aligned}
$$

$I[i][j]$ is the input of sub-activity/process, $P / A[i][j]$; $O[i][j]$ is the output of subactivity/process, $P / A[i][j] ; n$ is the number of $P / A[i]$ 's son-activity/process, $P / A[i][k]$.

External input and output of $P / A[i][j]$ are as follow:

$$
\begin{aligned}
& I^{\prime}[i][j]=I[i][j]-I[i][j] \cap \sum_{k=1, k \neq j}^{n} O[i][k] \\
& O^{\prime}[i][j]=O[i][j]-O[i][j] \cap \sum_{k=1, k \neq j}^{n} I[i][k]
\end{aligned}
$$

Then

$$
\begin{aligned}
& I[i]=\sum_{j=1}^{m} I[i][j] \\
& O[i]=\sum_{j=1}^{m} O{ }^{\prime}[i][j]
\end{aligned}
$$

In equation(6), $O[i]=g(I[i]$, ar [i] , ar [i] $\in A R$ [i]). If activity attribute state space, ar [i], could meet the need of transferring input into output, then

$$
O[i]=g^{\prime}(I[i])
$$

From equation (6),(10)',(11)', there is

$$
g^{\prime}(I[i])=\sum_{j=1}^{m} g^{\prime}\left(I^{\prime}[i][j]\right)
$$

Considering the coupling between input and output as well as the interaction among activity attributes, then 
An Integrated Modeling Method Supporting Product Development Process Optimization

$$
g(I[i])=\sum_{i=1}^{m} g(I[i][j]+f[i])
$$

f[i] represents the function of information transferring, managing and harmonizing among A[i]'s son-activities.

\section{DMP/A MODELING METHOD}

On the basis of PDP integrated modeling theory, we propose Product Development Process Modeling based on the Process/Action (DMP/A) method. The elements of DMP/A methods include activity/process block, link and joint (Figure 4). Process model is described by figure, table and embedded file. Here introduce briefly DMP/A method.

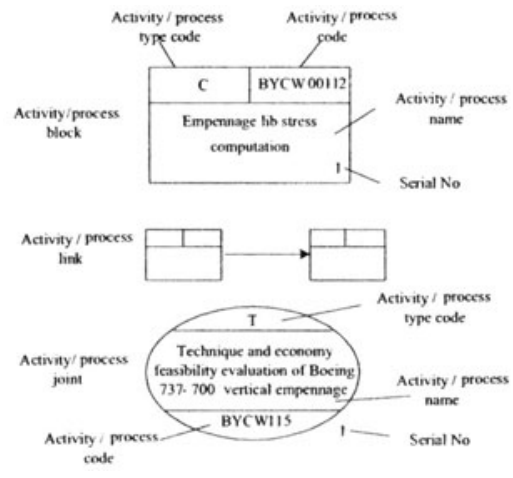

Figure 4. Activity/Process Block, Link and Joint

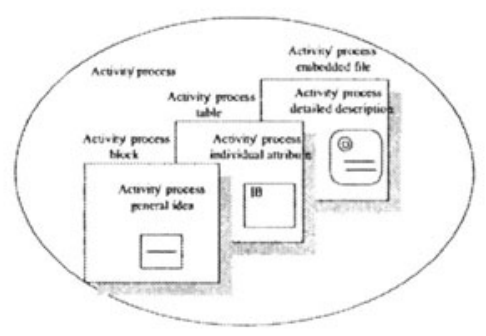

Figure 5. Relationship among Activity/Process Block, Table and Embedded File

\subsection{Activity/process Description}

DMP/A method uses activity/process block (joint), activity/process table and activity/process embedded file to describe activity/process. Activity/process block (joint) describes activity/process in general with visible figures; activity/process table describes activity/process resources, personnel, time and cost etc. by their individual character or attributes; activity/process embedded file is a more detailed description by explaining activity/process task, goal, rules and restrictions. Figure 5 shows the relationship among them.

\subsection{Activity/Process Link Description}

Activity/process link describes relation among activity/process in three ways: quality and quantity, direction and time. 
- Quality and quantity

Relation among activity/process is character with quality and quantity and could be described by figures, words or graphics document. Figures could be used to analyze quantitatively relation among activity/process, and words or graphics could be used to analyze qualitatively relation among activity/process. So, quantitative analysis and qualitatively analysis are combined.

- Direction

Product development system is ordered, which is displayed by the direction of interaction and relation among activity/process.

- Time

Time is a characteristic of relation among activity/process. Relation among activity/process exists during certain period and might disappear in other period. Relation is changing along with the time's changing.

The describing means of activity/process link include activity/process connecting line, link table and link embedded file. Activity/process connecting line uses arrow to express the direction of interaction and relation among activity/process. Link table and link embedded file describe the quality and quantity of relation among activity/process. Link table shows attributes of activity/process link, including time attribute. Link embedded file shows the content and other explanation of activity/process link. It is different with activity/process embedded file in that it includes not only the description file of task and goal of activity/process link, but also the description file of inputs and outputs of activity/process. These inputs and outputs usually could not be expressed by simple data type like integer or character string, but could only be stored as file, such as product blue print. Two types of activity/process link are displayed in Figure 6. Internal activity/process link represents the information or material flow within a father-activity/process, and external activity/process link is on the contrary.
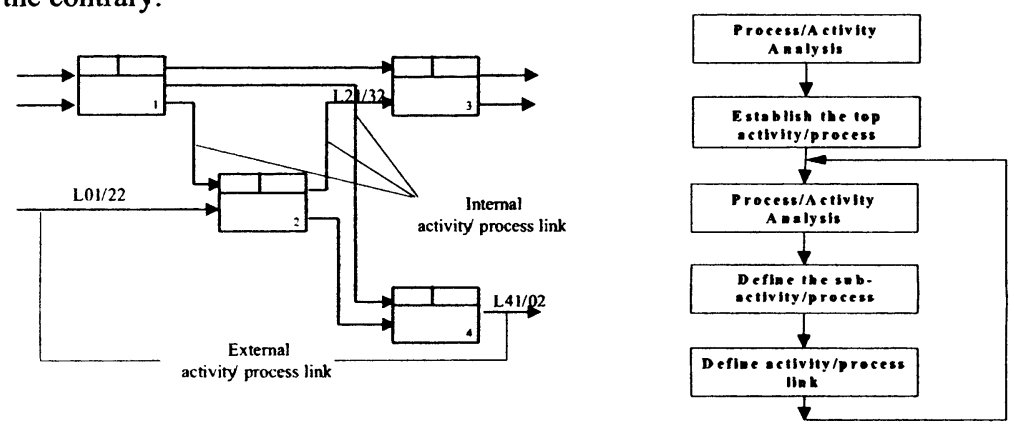

Figure 6. Internal and External Activity/Process Link Figure 7. Procedure of DMP/A

\subsection{Activity/Process Joint Description}

Joint is a special activity/process in DMP/A. It represents the general analysis or decision-making unit in PDP. Joint is displayed by ellipse (Figure 4). This ellipse is 
divided into three parts by two horizontal lines: the upper is filled with joint type code, the under with joint code and the middle with joint name. The joint serial No. is marked on the right under corner. Process routes may diverge or converge through the joint. Divergence and convergence are shown with activity/process connecting line of different direction.

The activity/process block and the joint use the uniform code, with the same code method. Usually, joint is not broken down. The joint table and embedded file is similar with the activity/process table and embedded file in structure and content. Compared with activity/process table, joint table is added with judging principle of process path. These judging principles may be text or formula, and they are the basis of choosing process path on joint. For example, 'if it passed the design examine' is the judging principle on joint 'design examine'. Further, judging principle could be divided into some rules. According to the information input and judging principle, a joint determines the process path.

\subsection{Process/Activity Module}

For different product or different parts from one product, object of process/activity dealt with may be differ in thousands ways, but their function and attributes in the attribute state space (ex. time, cost, etc.) are finite. The process/activity which have same or similar functions have the similar personnel constitute, resources type and input, output type. So, these process/activity and its personnel constitute, resources type and input, output type could be distilled for reuse. Then the deployment of product development process could use these standard modules, like the standard parts product, after deploying locally the process/activity, assembling the local process/activity to form the product development system.

Process/activity module is introduced like the toy bricks to construct a product development system. Every process/activity module has special relationship with its personnel constitute, resources type and input, output type, which are encapsulated into the module as well as the function. Through the process/activity module, the relationship among process/activity class and organize class, resource class, input and output could be established. Relating the process/activity with enterprise information system helps reorganize the product information and management information.

\subsection{Procedure of DMP/A Modeling (Figure 7)}

\section{Process/activity analysis}

Through process/activity analysis, concept of a certain field product development process is established, and process/activity type, process/activity function, personnel and resources needed in the product development are confirmed.

2. Establish the top process/activity

The top process/activity should be established firstly, and top process/activity table filled, with process/activity name, personnel, resources and inputs, outputs defined.

3. Process/activity breaking down 
Process/activity should be broken down into a series of sub-process/activity in the process/activity table. When there existed similar process/activity, it could be transferred and modified to form new process/activity table.

4. Define the sub-process/activity

The table of process/activity broken down should be filled, with process/activity name, personnel, resources and inputs, outputs be defined. Those process/activity that transfer process/activity modules, because the corresponding resources type, personnel type and inputs, outputs have been defined in the process/activity module warehouse, only need to choose from corresponding type of resources, personnel and inputs, outputs.

5. Define process/activity link

Draw the process/activity link, fill in its corresponding link table, define its name, code and product information code, etc.

Repeating step 3 to 5 , until every level of process/activity and process/activity link are established.

\section{CONCLUSIONS}

According to DMP/A method introduced in this paper, combined with the 863/CIMS projects, 'the Study of Double Integrated Framework/Platform and its Object-Oriented Application under Concurrent Engineering Overall Environment in CIMS' and 'Integrated Process Modeling Technology during Product Life Cycle', and the cooperation research project 'New Product Trial-Manufacturing Process Management System', a PDP management integrated application system, Design/PA, was established with the airplane's vertical empennage rib as research object. Through definition and description of process structure, activity/process attributes, activity/process inputs and outputs, Design/PA realized visibly the decomposition and programming of PDP, which could make better use of the process information and reduce the repetition in process programming. Moreover, Design/PA realized information integration based on activity/process and uniform information model. It provides a favorable environment for programming, supervising and managing PDP.

\section{REFERENCES}

1. W. Bernhard, Integrated Product and Process Management for Engineering Design Applications, Integrated Computer Aided Engineering. Volume 3, Number 1, pp.20-31, (1996).

2. Y. Xu, Study on Product Development Process Modeling. Ph.D Thesis, Northwestern Polytechnical University (2000).

3. M. Li, Study on Integrated Optimization Management of Product Development Process and its Key Technology. Ph.D Thesis, Northwestern Polytechnical University (2004). 